\title{
Correlation Study of Color Doppler Examination of Deep Veins of Both Lower Extremities Combined with Fibrinolysis System in Hypertension during Pregnancy
}

\author{
Chundong Qiu1, Haiyan Chen²*, Xiaohua Huang3", Xiaoling Kong1, Chong Liang4, Chunhong Qiu² \\ ${ }^{1}$ Department of Obstetrics, The First People's Hospital of Yulin City, Yulin, China \\ ${ }^{2}$ Department of Obstetrics, The Second People's Hospital of Yulin City, Yulin, China \\ ${ }^{3}$ Department of Clinical Laboratory, Yunyang Hospital of Traditional Chinese Medicine, Chongqing, China \\ ${ }^{4}$ Department of Clinical Laboratory, Red Cross Hospital of Yulin City, Yulin, China \\ Email: ^1012657363@qq.com, ^54076125@qq.com
}

How to cite this paper: Qiu, C.D., Chen, H.Y., Huang, X.H., Kong, X.L., Liang, C. and Qiu, C.H. (2020) Correlation Study of Color Doppler Examination of Deep Veins of Both Lower Extremities Combined with Fibrinolysis System in Hypertension during Pregnancy. Open Journal of Obstetrics and Gynecology, 10, 981-989.

https://doi.org/10.4236/ojog.2020.1080093

Received: July 9, 2020

Accepted: August 1, 2020

Published: August 4, 2020

Copyright $\odot 2020$ by author(s) and Scientific Research Publishing Inc. This work is licensed under the Creative Commons Attribution International License (CC BY 4.0).

http://creativecommons.org/licenses/by/4.0/ (c) (i) Open Access

\begin{abstract}
Objective: To investigate the correlation between deep static color Doppler ultrasound of both lower extremities combined with fibrinolytic system detection in pregnancy-induced hypertension. Methods: A total of 70 normal pregnant women, 70 pregnant women with hypertension during pregnancy, and 50 healthy non-pregnant women in the same period were selected as the research objects. The deep veins of both lower limbs were examined by Rili Erlangshen's color Doppler ultrasound diagnostic instrument (CDU), and the fibrinolysis system was tested by enzyme-linked immunosorbent assay (ELISA) method and automatic hemagglutination analyzer. Results: Compared with the non-pregnant group, the inner diameters of the left/right femoral vein (CFV) and popliteal vein (POV) in the normal pregnancy group and the pregnancy-induced hypertension group were significantly widened, and the peak blood flow spectrum was significantly reduced. The difference was statistically significant $(\mathrm{P}<0.01)$; FIB, PLG, t-PA, PAI, D-Dimer were significantly increased, the difference was statistically significant $(\mathrm{P}<0.01)$; Compared with the normal pregnancy group, the inner diameter of the left/right femoral vein (CFV) in the hypertensive pregnancy group was significantly wider than that in the normal pregnancy group, and the peak value of the left/right POV blood flow spectrum was significantly lower than that in the normal pregnancy group, the difference was statistically significant $(\mathrm{P}<0.01)$, while the left/right popliteal vein (POV) inner diameter, left/right CFV blood flow peak value did not change significantly from the normal pregnancy group,
\end{abstract}


and the difference was not statistically significant (P > 0.05); FIB, PLG, PAI, $\mathrm{D}$-Dimer of the hypertensive disease group during pregnancy significantly increased, the difference was statistically significant $(\mathrm{P}<0.01)$, while t-PA was not statistically significant $(\mathrm{P}>0.05)$. Conclusion: Normal pregnant women have a hypercoagulable state compared with healthy non-pregnant women, and pregnant women with hypertension during pregnancy are more likely to be in a pre-thrombotic state than pregnant women with normal pregnancy. Deep vein CDU examination of both lower extremities combined with blood fibrinolysis monitoring is of great value in the prevention and treatment of hypertension during pregnancy.

\section{Keywords}

Hypertension during Pregnancy, Prothrombotic State, Color Doppler Ultrasound, Fibrinolysis Index

\section{Introduction}

Hypertension during pregnancy is often in an abnormal hypercoagulable state. The body is often accompanied by hypoxia and injury of small blood vessel endothelial cells, as well as abnormalities in functions such as coagulation, fibrinolysis, and platelets. These abnormal pathological changes lead to the occurrence of prothrombotic state (PTS) in hypertensive disorders complicating pregnancy [1] [2]. The pre-thrombotic state of hypertension during pregnancy will also significantly increase the mortality of perinatal woman and child, which will have a significant negative impact on the survival and life of the majority of women and babies. With the comprehensive opening of China's second child policy, the number of pregnant women has increased significantly, and hypertension during pregnancy has increasingly attracted the attention of pregnant women [3] [4]. This study intends to explore the correlation between the results of deep venous color Doppler ultrasound examination combined with fibrinolysis system and hypertension in pregnancy. The results are reported below.

\section{Information and Methods}

\subsection{Research Objects and Groups}

\subsubsection{Grouping Standards and Requirements}

The diagnostic criteria for hypertension in pregnancy were based on the 8th edition of "Obstetrics and Gynecology" of the People's Medical Publishing House [5]. The pregnant group selected pregnant women with normal heart, liver, kidney, brain, blood vessels and coagulation function before pregnancy; In the non-pregnancy group, women with normal functions of heart, liver, kidney, brain and blood coagulation were selected, and they had not used procoagulant drugs such as contraceptives for half a year. All participants did not take drugs affecting heart, liver, kidney, brain function and coagulation function 1 week 
before venous blood drawing.

\subsubsection{Research Objects and Groups}

Retrospective analysis of 70 cases of normal pregnant women hospitalized in obstetrics and gynecology from June 2018 to December 2019 (normal pregnancy group), 70 cases of pregnant women with hypertension during pregnancy (pregnancy-induced hypertension group) and 50 cases of healthy non-pregnant women (normal non-pregnancy group), including 19 cases of preeclampsia (mild), 26 cases of preeclampsia (severe) and 25 cases of eclampsia in the hypertensive disease group of pregnancy, aged 22 to 39 years, average 28 years old, the gestational week is 32 - 42 weeks, average 36 weeks; the normal pregnancy group is 20 - 38 years old, average 27 years old, the gestational week is 32 - 41 weeks, average 37 weeks; 50 normal non-pregnant women, age 21 - 38 years old, average 27 years old.

\subsection{Instruments and Testing Methods}

\subsubsection{Apparatus and Method for Deep Vein Examination of Lower Extremities}

Using the Rili series (Erlangshen) color Doppler ultrasound diagnostic apparatus to perform deep vein examination of the lower extremities of the three groups of candidates, detect the inner diameter of the left and right femoral veins, popliteal veins and peak blood flow spectrum and other indicators to observe the presence of thrombosis form. Calibration of wake-up equipment is before inspection.

\subsubsection{Apparatus and Method for Detecting Blood Fibrinolysis Index}

t-PA is measured by American Pacific company EusA kit. The operation is determined according to the instructions. The rest of the items are tested by French STAGO-COMPACT hemagglutination instrument and the STAGO original kit is provided by Nanning Henghe Company; FIB uses steel ball coagulation method, D-Dimer determination using immunoturbidimetric method, PLG, PAI using chromogenic substrate method, t-PA determination using the quantitative method of enzyme-linked immunosorbent double antibody sandwich method (ELISA). All reagents are used within the validity period, and relevant indoor quality control is required before specimen testing.

\subsubsection{Blood Specimen Collection and Processing}

The venous blood of three groups of subjects was drawn into a vacuum anticoagulation tube containing 3.2\% sodium citrate, anticoagulated according to the ratio of 9:1, and the plasma was centrifuged at $2700 \mathrm{~g}$ for $10 \mathrm{~min}$ at room temperature to be tested.

\subsubsection{Statistical Processing}

SPSS19.0 statistical software was used for statistical analysis. The measurement data used two independent samples of $t$ test, and the count data used $\chi^{2}$ test or Fisher exact probability method. $\mathrm{P}<0.05$ was considered statistically significant. 


\section{Result}

\subsection{Compared with Non-Pregnancy Group}

FIB, PLG, t-PA, PAI, D-Dimer in normal pregnancy group and pregnancy-induced hypertension group were significantly increased, the difference was statistically significant $(\mathrm{P}<0.01)$; compared with normal pregnancy group, FIB, PLG, PAI, D-Dimer in pregnancy-induced hypertension group were significantly increased, the difference was statistically significant $(\mathrm{P}<0.01)$, t-PA was not statistically significant $(\mathrm{P}>0.05)$ (see Table 1 , Table 2 ).

\subsection{Comparison of the Results of Deep Vein Examination of Lower Extremities}

The inner diameters of the left and right CFV and POV in the normal pregnancy group and the pregnancy-induced hypertension group were significantly larger than those in the non-pregnancy group, and the peak blood flow spectrum was significantly reduced, the difference was statistically significant $(\mathrm{P}<0.01)$.

The diameter of the left/right femoral vein (CFV) in the hypertensive disorder pregnancy group was significantly larger than that in the normal pregnancy group, and the peak value of the left/right POV blood flow spectrum was significantly lower than that in the normal pregnancy group $(\mathrm{P}<0.01)$. However, the inner diameter of the left/right popliteal vein (POV) and the peak value of the left/right CFV blood flow were not significantly changed from the normal pregnancy group, and the difference was not statistically significant $(P>0.05)$ (see Table 3, Table 4).

Table 1. Test results of three groups of fibrinolytic system indexes ( $\pm s)$.

\begin{tabular}{ccccccc}
\hline Groups & Number of cases & FIB $(\mathrm{g} / \mathrm{L})$ & PLG $(\mathrm{U} / \mathrm{ml})$ & t-PA $(\mathrm{U} / \mathrm{ml})$ & PAI $(\mathrm{U} / \mathrm{ml})$ & D-Dimer $(\mathrm{ng} / \mathrm{L})$ \\
\hline Non-pregnant group & 50 & $2.26 \pm 0.18$ & $95.36 \pm 2.94$ & $1.30 \pm 0.06$ & $3.13 \pm 0.23$ & $0.22 \pm 0.02$ \\
Normal pregnancy group & 70 & $5.04 \pm 0.18^{*}$ & $119.71 \pm 3.41^{*}$ & $4.30 \pm 0.12^{*}$ & $6.54 \pm 0.23^{*}$ & $0.67 \pm 0.03^{*}$ \\
Pregnancy-induced hypertension group & 70 & $6.47 \pm 0.41^{* *}$ & $156.63 \pm 3.28^{* *}$ & $4.34 \pm 0.13^{*}$ & $10.70 \pm 1.04^{* *}$ & $1.44 \pm 0.10^{* *}$ \\
\hline
\end{tabular}

Note: 1) The pregnancy-induced hypertension group is the abbreviation of pregnancy-induced hypertension disease group; 2) Compared with non-pregnancy group, ${ }^{*} \mathrm{P}<0.01$; Compared with normal pregnancy group, $" \mathrm{P}<0.01$.

Table 2. T value and $\mathrm{P}$ value of three groups of fibrinolytic system indexes.

\begin{tabular}{cccccc}
\hline T value/P value & FIB $(\mathrm{g} / \mathrm{L})$ & PLG $(\mathrm{U} / \mathrm{ml})$ & $\mathrm{t}-\mathrm{PA}(\mathrm{U} / \mathrm{ml})$ & $\mathrm{PAI}(\mathrm{U} / \mathrm{ml})$ & D-Dimer $(\mathrm{ng} / \mathrm{L})$ \\
\hline T1 & 83.41 & 40.8 & 151.9 & 80.07 & 92.36 \\
T2 & 68.01 & 105.27 & 153.92 & 50.54 & 84.96 \\
T3 & 26.72 & 65.28 & 1.89 & 32.68 & 61.71 \\
P1 & $<0.001$ & $<0.001$ & $<0.001$ & $<0.001$ & $<0.001$ \\
P2 & $<0.001$ & $<0.001$ & $<0.001$ & $<0.001$ & $<0.001$ \\
P3 & $<0.001$ & $<0.001$ & $>0.05$ & $<0.001$ & $<0.001$ \\
\hline
\end{tabular}

Note: The $t$ and $\mathrm{P}$ values of normal pregnancy group and non-pregnancy group were T1 and P1. The $t$ and $\mathrm{P}$ values of the hypertensive disease group during pregnancy compared with the non-pregnant group were $\mathrm{T} 2$ and $\mathrm{P} 2$. The $\mathrm{t}$ and $\mathrm{P}$ values of the hypertensive disease group during pregnancy compared with the normal pregnancy group were $\mathrm{T} 3$ and $\mathrm{P} 3$. 
Table 3. Three groups of double lower limb CFV, POV inner diameter and blood flow spectrum peaking $(\mathrm{x} \pm \mathrm{s})$.

\begin{tabular}{ccccc}
\hline $\begin{array}{c}\text { Inner diameter of both } \\
\text { lower limbs/Peak blood } \\
\text { flow spectrum }\end{array}$ & $\begin{array}{c}\text { Left/Right } \\
\text { Left }\end{array}$ & $\begin{array}{c}\text { pregnancy-induced } \\
\text { hypertension group } \\
(70 \text { cases })\end{array}$ & $\begin{array}{c}1.37 \pm 0.09^{* *} \\
\text { normal pregnancy } \\
\text { group (70 cases) }\end{array}$ & $\begin{array}{c}\text { non-pregnancy } \\
\text { group (50 cases) }\end{array}$ \\
\hline CFV inner diameter $(\mathrm{cm})$ & Right & $1.34 \pm 0.11^{* *}$ & $1.09 \pm 0.10^{*}$ & $0.70 \pm 0.02$ \\
& Left & $7.94 \pm 1.83^{*}$ & $8.55 \pm 2.08^{*}$ & $0.70 \pm 0.03$ \\
CFV spectral peaks $(\mathrm{cm} / \mathrm{s})$ & Right & $7.97 \pm 1.70^{*}$ & $8.60 \pm 2.08^{*}$ & $17.39 \pm 1.77$ \\
& Left & $0.69 \pm 0.05^{*}$ & $0.68 \pm 0.03^{*}$ & $0.48 \pm 0.02$ \\
POV inner diameter $(\mathrm{cm})$ & Right & $0.69 \pm 0.04^{*}$ & $0.68 \pm 0.02^{*}$ & $0.48 \pm 0.03$ \\
& Left & $5.04 \pm 0.79^{*}$ & $7.09 \pm 1.28^{*}$ & $12.45 \pm 1.97$ \\
\hline
\end{tabular}

Note: Compared with non-pregnant group ${ }^{\star} \mathrm{P}<0.01$; compared with normal pregnant group "P $<0.01$.

Table 4. Comparison of $\mathrm{T}$ value and $\mathrm{P}$ value of CFV inner diameter, POV inner diameter and blood flow spectrum peak value among the three groups.

\begin{tabular}{ccccccccc}
\hline \multirow{2}{*}{$\begin{array}{c}\text { T value/P } \\
\text { value }\end{array}$} & \multicolumn{2}{c}{ CFV Inner diameter } & \multicolumn{2}{c}{ CFV Spectral peak } & \multicolumn{2}{c}{ POV Inner diameter } & \multicolumn{2}{c}{ POV Spectral peak } \\
\cline { 2 - 9 } & Left & Right & Left & Right & Left & Right & Left & Right \\
\hline T1 & 28.81 & 29.31 & 22.6 & 23.35 & 133.63 & 30.6 & 18.03 & 17.09 \\
T2 & 49.12 & 39.64 & 26.42 & 30.94 & 29.43 & 31.34 & 28.46 & 27.17 \\
T3 & 16.99 & 14.14 & 1.84 & 1.96 & 1.44 & 1.87 & 11.37 & 10.36 \\
P1 & $<0.001$ & $<0.001$ & $<0.001$ & $<0.001$ & $<0.001$ & $<0.001$ & $<0.001$ & $<0.001$ \\
P2 & $<0.001$ & $<0.001$ & $<0.001$ & $<0.001$ & $<0.001$ & $<0.001$ & $<0.001$ & $<0.001$ \\
P3 & $<0.001$ & $<0.001$ & $>0.05$ & $>0.05$ & $>0.05$ & $>0.05$ & $<0.001$ & $<0.001$ \\
\hline
\end{tabular}

Note: 1) The comparison of $t$ value and $p$ value of normal pregnancy group and non-pregnancy group are $\mathrm{T} 1$ and $\mathrm{P} 1$; The comparison of $\mathrm{t}$ value and $\mathrm{p}$ value of pregnancy hypertensive disease group and non-pregnancy group are $\mathrm{T} 2$ and $\mathrm{P} 2$; The comparison of $\mathrm{t}$ value and $\mathrm{p}$ value of pregnancy hypertensive disease group and normal pregnancy group are $\mathrm{T} 3$ and $\mathrm{P} 3$.

\section{Discussion}

Hypertensive disorder complicating pregnancy is a common complication in modern perinatal medicine and is one of the main factors of maternal and infant death. Hypertensive disorders complicating pregnancy are often accompanied by coagulation, fibrinolysis, platelet and vascular abnormalities. These changes are closely related to the occurrence and development of prothrombotic state (PTS) in hypertensive disorders complicating pregnancy [1]-[6].

Recent years, some scholars have proposed the laboratory index reflecting the activity of fibrinolytic system as the PTS specific experimental diagnostic index, while the detection value of deep vein diameter of both lower limbs and the change of its peak value of blood flow spectrum, hemodynamic index and blood screening index are basically consistent, which can be used as a reference for the diagnosis of PTS [7] [8] [9] [10]. 
FIB is one of the important factors of thrombosis, and the FIB amount in blood is positively correlated with the hypercoagulability of the body; the higher the amount, the easier it is to form a thrombus. Plasminogen only plays an active role when it is converted to plasmin, and tissue plasminogen activator (t-PA) is the key enzyme for the conversion of plasminogen to plasmin, which can cleave fibrin Protolyze and hydrolyze a variety of activated coagulation factors, so when the amount of t-PA increases, the amount of plasmin also increases, and eventually the body's fibrinolytic activity is enhanced; while the role of PAI is opposite, PAI and t-PA form a complex which causes t-PA to lose activity [11] [12]. The results of this study showed that the levels of FIB and PLG in the normal pregnancy group and the pregnancy-induced hypertension disease group were significantly higher than those in the non-pregnancy group, and the pregnancy-induced hypertension disease group was significantly higher than the normal pregnancy group, and the differences between the groups were statistically significant, suggesting that normal pregnant women have increased FIB formation in the blood, the body is in a physiological hypercoagulable state, and patients with hypertension during pregnancy are in a pathological hypercoagulable state. The levels of t-PA and PAI in the normal pregnancy group and the pregnancy-induced hypertension disease group were higher than those in the non-pregnancy group. Because the increase in t-PA in the normal pregnancy group was greater than the increase in PAI, the fibrinolytic activity increased and the body was in high-level dynamic balance between coagulation and fibrinolysis. The levels of t-PA and PAI in normal pregnancy group and pregnancy-induced hypertension group were higher than those in non-pregnancy group; the increase of t-PA was higher than PAI in normal pregnancy group, which resulted in the increase of fibrinolytic activity, and the body was in high-level dynamic balance between coagulation and fibrinolysis. There was no significant difference in t-PA between normal pregnancy group and pregnancy-induced hypertension disease group, while PAI was further increased in patients with pregnancy-induced hypertension disease, and the increase of PAI exceeded the increase of $\mathrm{t}-\mathrm{PA}$, which led to the high-level dynamic balance of coagulation and fibrinolysis being broken again, resulting in blood hypercoagulability of the body further aggravated. The specific degradation product of fibrin is D-Dimer; the increased level of D-Dimer indicates that the body's fibrinolysis system is activated and reflects the secondary fibrinolysis enhancement; it can sensitively reflect the state of coagulation and fibrinolysis in the body.

The results of this study showed that the plasma D-Dimer levels of pregnant women in normal pregnancy were higher than those in healthy non-pregnant women and the difference was statistically significant, indicating that pregnant women were in a physiological hypercoagulable state, with increased fibrinolytic activity and increased fibrin degradation products (D-Dimer). The results of this study also showed that the level of D-Dimer in patients with hypertension during pregnancy was significantly higher than that in normal pregnant women, which was related to the ischemia, hypoxia, severe damage of vascular endothe- 
lial cell structure and villon necrosis of placental capillaries in patients with hypertensive disorder complicating pregnancy; these changes prompted the release of a large number of tissue thromboplastin, resulting in the formation of intravascular microthrombus; at the same time, secondary fibrinolysis was also activated, which led to a significant increase in D-Dimer level. When the level of D-Dimer in patients with hypertension during pregnancy is significantly increased, it suggests microthrombosis and there may be asymptomatic chronic DIC [13] [14].

The results of ultrasonic examination showed that the deep diameter of both lower limbs was significantly larger than that of non-pregnant pregnant women, and the peak value of blood flow spectrum was significantly smaller than that of non-pregnant pregnant women; it suggested that normal pregnant women's lower limb vein diameter widened, vein velocity slowed down, and thrombosis was easy to occur; the results of deep color Doppler ultrasound in both lower extremities were basically consistent with the results of blood fibrinolysis index, indicating that pregnant women were more likely to be in the pre-thrombotic state than healthy non-pregnant women. The FIB, PLG, PAI, D-Dimers of hypertensive disorder complicating pregnancy were significantly higher than those of normal pregnant women, while the venous diameter of most lower limbs was significantly wider than that of normal pregnant women, and the peak value of some blood flow spectrum was significantly lower, indicating that patients with hypertensive disorder complicating pregnancy were more likely to be in PTS than normal pregnant women.

\section{Conclusion}

The examination results of this study showed that the detection value of deep vein diameter of lower extremities and the peak value of deep vein blood flow spectrum of lower extremities were basically consistent with the changes of fibrinolysis system detection index, which was consistent with the relevant research results [8]. However, the vascular ultrasound imaging diagnosis method is expensive and the imaging false negative causes the delayed diagnosis and other problems. At present, it is generally believed that fibrinolytic system detection index can be used as a sensitive index for screening thromboembolic diseases during pregnancy and postpartum. Under the condition of economic permission, the author thinks that deep vein examination of lower extremity combined with fibrinolytic system index detection will be more helpful to improve the diagnosis rate of prothrombotic state of hypertensive disorder complicating pregnancy, so as to detect early and intervene early, thus reducing the mortality rate of perinatal pregnant women and perinatal children.

\section{Limitations of the Study}

This study mainly observes a limited number of cases, which has certain limitations, and the number of cases can be expanded to obtain more powerful evi- 
dence. In addition, the follow-up time of this study is relatively long, and the patient's compliance with follow-up has a certain complexity, which has a certain impact on the results.

\section{Acknowledgements}

This project was approved with the strong support from functional departments of the hospital and the help from colleagues. In the implementation process, this project also received the strong help and support from colleagues, patients and patients' families. I would like to express my heartfelt thanks to them for their support and help! I wish them good health, good luck in their work and all the best!

\section{Fund Project}

Scientific Research and Technology Development Project of Yulin City, Guangxi (Yushi Branch 20171624).

\section{Conflicts of Interest}

The authors declare no conflicts of interest regarding the publication of this paper.

\section{References}

[1] Chinese Medical Association Obstetrics and Gynecology Branch of Hypertensive Disorder Complicating Pregnancy (2015) Guidelines for the Diagnosis and Treatment of Hypertensive Disorder Complicating Pregnancy (2015). Chinese Journal of Obstetrics and Gynecology, 50, 721-728.

[2] Yang, Z. and Zhang, W. (2019) Early Identification of Hypertensive Disorder Complicating Pregnancy (1). Chinese Journal of Obstetrics and Gynecology, 54, 501-502.

[3] Chinese Academy of Medicine Obstetrics and Gynecology Branch Pregnancy Hypertension Disease Group (2012) Guidelines for the Diagnosis and Treatment of Pregnancy-Induced Hypertension Diseases (2012 Edition). Chinese Journal of $\mathrm{Ob}$ stetrics and Gynecology, 47, 476-480.

[4] Chinese Medical Association Obstetrics and Gynecology Branch Pregnancy Hypertensive Diseases Group (2019) Expert Consensus on the Management of Pre-Pregnancy, Pregnancy and Childbirth of Elderly Women (2019). Chinese Journal of Obstetrics and Gynecology, 54, 24-26.

[5] Xie, X. and Gou, W., Eds. (2018) Obstetrics and Gynecology. 8th Edition, People's Medical Publishing House, Beijing.

[6] Zheng, X., Zheng, X. and Wu, C. (2019) Analysis of Risk Factors of Hypertension in Pregnant Women during Pregnancy. Journal of Guangxi Medical University, 36, 943-947.

[7] Xu, J., Zhang, L., Wang, Y. and Han, Y. (2019) Clinical Effect of Hyperbaric Oxygen Adjuvant Therapy on Patients with Pregnancy Induced Hypertension and Its Effect on Partial Thrombin Function. Chinese Journal of Nautical Medicine and Hyperbaric Medicine, 26, 246-248.

[8] Hong, X. and Xi, J. (2019) Ultrasound Detection of Uterine Artery Blood Flow Combined with Mean Arterial Pressure in the Second Trimester for Screening the 
Effect of Pregnancy on Hypertension in Pregnant Women. Chinese Maternal and Child Health Care, 34, 4083-4086.

[9] Zheng, M. Hou, L., Li, B. and Wang, T. (2019) Research Progress of Color Doppler Ultrasound in Predicting Hypertension during Pregnancy. Chinese Maternal and Child Health Care, 34, 5563-5565.

[10] Luo, X. and Wang, T. (2019) Key Points of Clinical Management Guidelines for Obstetricians and Gynecologists ACOG Hypertensive Disorder Complicating Pregnancy in 2019. Journal of Practical Obstetrics and Gynecology, 35, 259-262.

[11] Zhai, Y. and Qi, Y. (2019) Clinical Significance of Peripheral Blood D-Dimer and Uterine Artery Blood Flow Monitoring in the Evaluation of Pregnancy-Induced Hypertension. Chinese Journal of Maternal and Child Health Care, 34, 1045-1047.

[12] Li, J. and Shen, X. (2019) Analysis of the Coagulation Function of Pregnant Women with Hypertension during Pregnancy and Its Correlation with Deep Vein Thrombosis. Chinese Maternal and Child Health Care, 34, 1259-1261.

[13] Cheng, X., Sun, B. and Su, Z. (2019) Research Progress of Thrombomodulin and Hypertension during Pregnancy. Chinese Journal of Maternal and Child Health Care, 34, 4353-4355.

[14] Mao, L. (2019) Analysis of the Detection Results of D-Dimer and Coagulation Indexes in Patients with Hypertension during Pregnancy. China Primary Medicine, 26, 2953-2956. 